\title{
On a convergent power series method to price defaultable bonds in a Vasicek-CIR model*
}

\author{
Fabio Antonelli $^{\dagger} \quad$ Alessandro Ramponi $^{\ddagger} \quad$ Sergio Scarlatti $^{\S}$
}

\begin{abstract}
In this paper, we prove that the price of a defaultable bond, under a Vasicek short rate dynamics coupled with a Cox-Ingersoll-Ross default intensity model, is a real analytic function, in a neighborhood of the origin, of the correlation parameter between the Brownian motions driving the processes, used to express the dependence between the short rate and the default intensity of the bond issuer. Employing conditioning and a change of numéraire technique, we obtain a manageable representation of the bond price in this non-affine model which allows us to control its derivatives and assess the convergence of the series. By truncating the expansion at the second order, a quadratic approximation formula for the price is then provided. Finally, practical applications of the result are highlighted by performing a numerical comparison with alternative pricing methodologies.
\end{abstract}

Keywords: credit risk; defaultable bond pricing; non-affine models; analytical functions; hazard process; change of numéraire.

MSC2020 subject classifications: 91G30; 91G60; $26 \mathrm{E} 05$.

Submitted to ECP on September 30, 2021, final version accepted on February 22, 2022.

\section{Introduction}

It has always been known that credit risk is one of the most important aspects of fixed income markets, for this reason bond pricing models must incorporate it in a meaningful way allowing, at the same time, for fast and efficient calibration of the model parameters to market data. In the last twenty years, two alternative approaches have been recognized to be capable to fulfill these requirements of coherence and applicability: the structural model and the intensity model, each one having its own advantages and limits. They are based on different assumptions, but attempts have been made to try to reconcile them ([15], [13], see also [12]). In this work, we fully pursue the second approach, while we refer to Merton's original paper [25] as the main source for the first one and to the classical books [7] and [23] for its subsequent large developments. The literature on the intensity-based approach, or reduced-form model, is also quite vast and the previous mentioned books (and refs therein) remain extremely good sources, however historically the main ideas were introduced by Jarrow and Turnbull [19] and

\footnotetext{
*The Authors F. Antonelli and A. Ramponi are members of INdAM-GNAMPA.

${ }^{\dagger}$ University of L'Aquila. E-mail: fabio. antonel li@univaq.it

${ }^{\ddagger}$ Dept. Economics and Finance, University of Roma - Tor Vergata. E-mail: alessandro. ramponi@uniroma2. it

${ }^{\S}$ Dept. Economics and Finance, University of Roma - Tor Vergata. E-mail: sergio.scarlatti@uniroma2.it
} 
then by Madan and Unal [26] and Lando [22]. In the reduced form model, the issuer may default at any time $\tau$ before the bond maturity. Moreover default comes unexpected, as experienced by many investors: it is an unpredictable event determined also by exogenous factors, and contrary to the structural approach it cannot be foreseen by monitoring the financial state of the bond issuer. On the mathematical side, the crucial assumption is the existence of the density for the conditional distribution of the random time $\tau$ given the smaller filtration containing all the market information, which leads to defining a hazard process with an intensity (see [8]). It is then common practice to calibrate conditional defaulting probabilities by using CDS spreads observed in the swap market, the ease of this calibration procedure being often considered a plus of the approach. However, in this respect, the possibility of hybrid models, such as [12], should be considered (see e.g. [5] where CDS pricing is discussed), since they might offer the advantage of being able to incorporate an analysis of credit implied volatilities, as recently done in [27] for the structural approach.

When considering an arbitrage free market where all securities are priced by using some short-rate process $\left(r_{t}\right)$ and a martingale measure $\mathbb{P}$, an important feature of the intensity based approach (strongly emphasized in the highly influential paper by Duffie and Singleton [16]) is that the defaultable securities prices can be obtained in a "defaultfree manner", by replacing the short rate process with the "default-adjusted" short-rate process given by the sum of $\left(r_{t}\right)$ and the intensity process $\left(\lambda_{t}\right)$, multiplied by the expected fractional loss $\left(L_{t}\right)$. When restricting to affine modeling of default-free bond prices, a simple consequence of the Duffie and Singleton observation is that if the default-adjusted short rate process remains in the affine class, then also defaultable bond prices can be computed by the same procedure used for the default-free case, a highly desirable feature.

In the present paper, we study a market where the default-adjusted short rate process falls out of the affine class and so the previous implication breaks down. This easily happens, when assuming that the short rate follows a Vasicek dynamics, while the intensity follows a CIR dynamics, with correlated driving Brownian motions. It is important to notice that this market model is not artificial, indeed it arises naturally, considering the current long period of zero or negative interest rates and that an intensity process must be necessarily positive to be interpreted as a (infinitesimal) defaulting probability (refer to [20] for a similar choice). It is readily seen that in case of non-zero correlation the system interest rate/intensity is not jointly affine, by consequence its analytical tractability becomes challenging since the usual pricing methods based on Fourier transforms are ruled out, leaving Monte Carlo simulations as the only actual computational tool. Although loss of affinity might look an unpleasant feature, one should be aware that non-affine models arise quite naturally also in the pricing of options under stochastic volatility, where they were found to be even more realistic than their affine counterparts (see e.g. [14],[18]).

From now on, we denote by $P^{d}(t, T)$ the current price of a defaultable bond ( $T$ being the maturity) with zero fractional recovery. This price is going to depend on several parameters appearing in the equations describing the evolution of the state variables, the interest rate and the intensity. Typically, these parameters have small values, in particular correlation, which cannot exceed the unit in absolute value. This opens up the possibility to evaluate the price $P^{d}(t, T)$, employing a power series expansion in the correlation parameter around the origin. If this series converges to the actual price, one can rightfully use it to derive an approximate formula up to any desired order of accuracy. At computational level this might provide a much faster evaluation than by MC methods, but with comparable accuracy.

Here, we denote by $\rho$ the correlation coefficient between the Brownian motions 
driving the state processes, and we prove that the defaultable bond price, $P^{d}(t, T)$ is an analytic function of $\rho$ in a neighborhood of the origin. To do so, we first condition the payoff to the filtration generated by the Brownian motion driving the intensity process, enucleating two functions of $\rho$, whose derivatives can be explicitly estimated. The estimates are sufficient to ensure the convergence of the series. To identify the series coefficients, we use a change of numéraire technique to isolate the contribution given by the intensity, that we can compute exploiting the affine properties of the CIR process. Finally, we use the second-order Taylor polynomial to write an approximation formula for the price.

In general, proving the convergence of the price's power series is very hard, and it strongly depends on the type of contract one considers. Once the price functional representation is written, the main issue is the estimation of its derivatives, that we could obtain in our case. Employing estimates of completely different type, similar results were proven in [4] under constant interest rate for a forward contract, and in [2] and [3], for option pricing, so providing, the same as here, approximation formulas with much shorter computational times than Monte Carlo simulations.

Price approximations via power expansion are not a novelty in the literature, often developed with respect to different parameters such as the volatility of the underlying asset (see for instance [1]). The advantage of our parameter choice resides in the fact that expanding at zero correlation often allows exploiting semi-explicit expressions under independence of the driving processes. We finally remark that our methodology extends quite easily to include more factors in the model, as shown in [4].

The paper is organized as follows. In Section 2, we present the market model, and via a conditioning technique, we show that the bond price is an analytic function of the correlation coefficient. In Section 3, we identify the series coefficients, by using the Girsanov theorem that makes it possible to employ the results of classical bond pricing theory to evaluate them. In Section 4, we consider a second-order approximation of the price, and we run a numerical analysis comparing it with the price evaluations obtained by Monte-Carlo simulations (MC method) and by a numerical method proposed in [17] by Grzelak and Oosterlee consisting in approximating a non affine system with a closely related affine one (GO method). The results show that our approximation has computational times comparable to those of the GO method, without losing accuracy (both methods being superior to MC). However, the main strength of our approach lies in the possibility to improve the error estimate by increasing the order of the approximation, by virtue of the convergence result.

\section{The Vasicek-CIR market model: the main result}

We consider a finite time interval $[0, T]$ and a complete probability space $(\Omega, \mathcal{F}, \mathbb{P})$, endowed with a filtration $\left\{\mathcal{F}_{t}\right\}_{t \in[0, T]}$, augmented with the $\mathbb{P}$ - null sets and made right continuous. We assume that all processes have a càdlàg version.

We are interested in pricing a defaultable zero-coupon bond, under the assumption that the short rate process $\left(r_{t}\right)$ follows a Vasicek model

$$
r_{t}=r+k \int_{0}^{t}\left(\theta-r_{s}\right) d s+\sigma B_{t}, \quad 0 \leq t \leq T,
$$

where $k, \sigma>0, r, \theta \in \mathbb{R}$, and $B$ is an $\mathcal{F}_{t}-$ Brownian motion. The probability $\mathbb{P}$ is a risk-neutral measure determined by the calibration of the market model.

The issuer of the bond might default at a random time $\tau$, which is not necessarily an $\mathcal{F}_{t}-$ stopping time. As we mentioned in the introduction, we are going to use the 
reduced-form approach, first progressively enlarging the filtration $\mathcal{F}_{t}$ by

$$
\mathcal{G}_{t}=\mathcal{F}_{t} \vee \sigma\left(\mathbf{1}_{\{\tau \leq s\}}, s \leq t\right) \quad \text { (the smallest filtration making } \tau \text { a stopping time), }
$$

then assuming we can represent the conditional distribution function $\mathbb{P}\left(\tau \leq t \mid \mathcal{F}_{t}\right)$ by a hazard process with intensity $\lambda_{t}$. We remark that here we are abusing a little the notation by keeping the same notation $\mathbb{P}$ for the extension of the probability to the $\mathcal{G}-$ filtration.

Then, by exploiting the well known Key Lemma and its extensions (see for instance [7], and [9]), we may write the price of a defaultable bond as

$$
P^{d}(t, T)=\mathbb{E}\left(\mathrm{e}^{-\int_{t}^{T} r_{s} d s} 1_{\{\tau>t\}} \mid \mathcal{F}_{t}\right)=1_{\{\tau>t\}} \mathbb{E}\left(\mathrm{e}^{-\int_{t}^{T}\left(r_{s}+\lambda_{s}\right) d s} \mid \mathcal{F}_{t}\right) .
$$

We also remark the payoff's square integrability guarantees that the prices remain martingales also in the larger $\mathcal{G}$ - filtration.

In general, we expect the processes $\left(r_{t}\right)$ and $\left(\lambda_{t}\right)$ to be correlated, as the interest rate might influence the issuer's solvability. To model this dependence, one can make several choices; a possibility is to take $\left(\lambda_{t}\right)$ as a function of the rate with the possible addition of an independent component (see [16]). We find this choice a little restrictive, and we represent the intensity by an additional diffusion, a CIR process

$$
\lambda_{t}=\lambda+\alpha \int_{0}^{t}\left(\beta-\lambda_{s}\right) d s+\gamma \int_{0}^{t} \sqrt{\lambda_{s}} d B_{s}^{1},
$$

with positive constants $\lambda, \alpha, \beta, \gamma$, and Feller's condition, $2 \alpha \beta>\gamma^{2}$, satisfied so that the process stays almost surely positive. The process $B^{1}$ is a Brownian motion correlated with $B$, such that $\left\langle B, B^{1}\right\rangle_{t}=\rho t$, with $\rho \in(-1,1)$, so possibly constructing an appropriate probability space, we may assume the pair $\left(r_{t}, \lambda_{t}\right)$ satisfies

$$
\begin{aligned}
& r_{t}=r+k \int_{0}^{t}\left(\theta-r_{s}\right) d s+\sigma\left[\rho B_{t}^{1}+\sqrt{1-\rho^{2}} B_{t}^{2}\right] \\
& \lambda_{t}=\lambda+\alpha \int_{0}^{t}\left(\beta-\lambda_{s}\right) d s+\gamma \int_{0}^{t} \sqrt{\lambda_{s}} d B_{s}^{1},
\end{aligned}
$$

where $B^{1}$ and $B^{2}$ are independent Brownian motions.

If the short rate and the intensity processes are independent, then (2.2) reduces to the product of the Vasicek and CIR bond prices $\left(\left(r_{t}\right)\right.$ and $\left(\lambda_{t}\right)$ being the respective rates),

$$
P^{d}(t, T)=P^{V}(t, T) P^{C I R}(t, T) .
$$

The processes $\left(r_{t}\right),\left(\lambda_{t}\right)$ are singularly affine, hence under independence, we have an explicit expression of each factor. Unfortunately, when $\rho \neq 0$, factorization (2.5) is no longer possible, the system (2.4) is not affine, and there is no hope to achieve an explicit evaluation of (2.2), applying Fourier transform techniques.

On the other hand, system (2.4) is Markovian, whence evaluation (2.2) is a Borel function of the state variables, which also depends on the correlation parameter $\rho$. Therefore, we may write

$$
P^{d}(t, T) \equiv P^{d}(t, r, \lambda, T ; \rho)=\mathbb{E}\left(\mathrm{e}^{-\int_{t}^{T} \lambda_{u} d u} \mathrm{e}^{-\int_{t}^{T} r_{u} d u} \mid r_{t}=r, \lambda_{t}=\lambda\right) .
$$

Remark 2.1. By exploiting Feynman-Kaĉ's formula within this market model, it turns out that $P^{d}(t, r, \lambda, T ; \rho)$ solves the following two-dimensional parabolic problem

$$
\left\{\begin{array}{l}
\frac{\partial u}{\partial t}(t, r, \lambda)+\mathcal{L}^{\rho} u(t, r, \lambda)=0 \quad(t, r, \lambda) \in[0, T) \times \mathbb{R} \times(0,+\infty) \\
u(T, r, \lambda)=1 \quad(r, \lambda) \in \mathbb{R} \times(0,+\infty),
\end{array}\right.
$$


with $\mathcal{L}^{\rho}:=\mathcal{L}^{0}+\mathcal{A}$, where

$$
\begin{aligned}
\mathcal{L}^{\mathbf{0}} & =\left(\frac{\sigma^{2}}{2} \frac{\partial^{2}}{\partial r^{2}}+\kappa(\theta-r) \frac{\partial}{\partial r}-r\right)+\left(\frac{\gamma^{2} \lambda}{2} \frac{\partial^{2}}{\partial \lambda^{2}}+\alpha(\beta-\lambda) \frac{\partial}{\partial \lambda}-\lambda\right) \\
\mathcal{A} & =\rho \sigma \gamma \sqrt{\lambda} \frac{\partial^{2}}{\partial r \partial \lambda} .
\end{aligned}
$$

When $\rho=0$ equation (2.7) can be explicitly solved by separating the variables.This confirms that it might be promising to develop the general solution in power series of $\rho$ around 0 .

In the following Theorem 2.3, we are going to show that $P^{d}(t, r, \lambda, T ; \cdot)$ is a $C^{\infty}$ function of $\rho$ and that, for each fixed $t, r, T$, the Taylor series

$$
\sum_{n=0}^{\infty} \frac{1}{n !} \frac{\partial^{n} P^{d}}{\partial \rho^{n}}(t, r, T ; 0) \rho^{n}
$$

converges to (2.6) in an open interval around $\rho=0$. Without loss of generality, we prove this result at $t=0$, as it is sufficient to substitute $T$ with $T-t$ in the general case.

We first provide an alternative representation for (2.6).

Lemma 2.2. Given the model (2.4), we have

$$
P^{d}(0, r, \lambda, T ; \rho)=\mathrm{e}^{-\theta T-(r-\theta) \frac{1-\mathrm{e}^{-k T}}{k}} F(\rho) G(\rho),
$$

where

$$
\begin{aligned}
& F(\rho)=\mathbb{E}\left(\mathrm{e}^{-\frac{\sigma}{k} \sqrt{1-\rho^{2}} \int_{0}^{T}\left[1-\mathrm{e}^{-k(T-s)}\right] d B_{s}^{2}}\right)=\mathrm{e}^{\frac{\sigma^{2}}{2 k^{2}}\left(1-\rho^{2}\right) \int_{0}^{T}\left[1-\mathrm{e}^{-k(T-s)}\right]^{2} d s}, \\
& G(\rho)=\mathbb{E}\left(\mathrm{e}^{-\int_{0}^{T} \lambda_{u} d u} \mathrm{e}^{-\frac{\sigma}{k} \rho \int_{0}^{T}\left[1-\mathrm{e}^{-k(T-s)}\right] d B_{s}^{1}}\right) .
\end{aligned}
$$

Proof. We recall that the explicit solution of (2.1) at time $t=T$ is

$$
r_{T}=\theta+(r-\theta) \mathrm{e}^{-k T}+\sigma \mathrm{e}^{-k T} \int_{0}^{T} \mathrm{e}^{k s} d B_{s}, \quad B_{s}=\rho B_{s}^{1}+\sqrt{1-\rho^{2}} B_{s}^{2},
$$

whence, by using the first equation in (2.4), we may derive

$$
\int_{0}^{T} r_{t} d t=\theta T+\frac{r-r_{T}+\sigma B_{T}}{k}=\theta T+(r-\theta) \frac{1-\mathrm{e}^{-k T}}{k}+\sigma \int_{0}^{T} \frac{1-\mathrm{e}^{-k(T-t)}}{k} d B_{t} .
$$

We have

$$
P^{d}(0, r, \lambda, T ; \rho)=\mathbb{E}\left(\mathrm{e}^{-\int_{0}^{T} \lambda_{u} d u} \mathrm{e}^{-\theta T-(r-\theta) \frac{1-\mathrm{e}^{-k T}}{k}-\sigma \int_{0}^{T} \frac{1-\mathrm{e}^{-k(T-t)}}{k} d\left(\rho B_{t}^{1}+\sqrt{1-\rho^{2}} B_{t}^{2}\right)}\right) .
$$

By conditioning the expectation argument to the $\sigma$-algebra $\mathcal{F}_{u}^{1}=\sigma\left(\left\{B_{s}^{1}: 0 \leq s \leq u\right\}\right)$, we obtain

$$
\begin{aligned}
& P^{d}(0, r, \lambda, T ; \rho)=\mathrm{e}^{-\theta T-(r-\theta) \frac{1-\mathrm{e}^{-k T}}{k}} \\
& \times \mathbb{E}\left[\mathrm{e}^{-\int_{0}^{T} \lambda_{u} d u-\frac{\sigma \rho}{k} \int_{0}^{T}\left[1-\mathrm{e}^{-k(T-t)}\right] d B_{t}^{1}} \mathbb{E}\left(\mathrm{e}^{-\frac{\sigma \sqrt{1-\rho^{2}}}{k}} \int_{0}^{T}\left[1-\mathrm{e}^{-k(T-t)}\right] d B_{t}^{2} \mid \mathcal{F}_{T}^{1}\right)\right]
\end{aligned}
$$

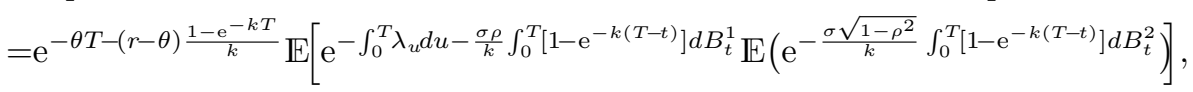

because of the independence of the two Brownian motions. The inner expectation is $F(\rho)$, and the last equality in (2.12) comes from the Gaussian distribution of the stochastic integral.

We finally present our main result. 
Theorem 2.3. Given the model (2.4), under Feller's condition, $P^{d}(0, r, \lambda, T ; \rho)$ given by (2.11) is a real-analytic function of $\rho$ in $(-1,1)$.

Proof. For the sake of simplicity, we denote by

$$
S_{t}=\mathrm{e}^{-\int_{0}^{t} \lambda_{u} d u}, \quad Z_{t}^{i}=\frac{\sigma}{k} \int_{0}^{t}\left(1-\mathrm{e}^{-k(t-s)}\right) d B_{s}^{i} \sim \mathcal{N}\left(0, \frac{\sigma^{2}}{k^{2}} \int_{0}^{t}\left(1-\mathrm{e}^{-k(t-s)}\right)^{2} d s\right), \quad i=1,2 .
$$

From (2.12), we have that the function $F(\rho)$ is a real analytic function of $\rho$, and we may write

$$
G(\rho)=\mathbb{E}\left(S_{T} e^{-\rho Z_{T}^{1}}\right) .
$$

By Feller's condition $S_{T} \leq 1$, therefore we have

$$
\begin{aligned}
\left|\frac{\partial^{n}}{\partial \rho^{n}} G(\rho)\right| & =\mid \mathbb{E}\left(S_{T} \frac{\partial^{n}}{\partial \rho^{n}}\left(\mathrm{e}^{-\rho Z_{T}^{1}}\right)|=| \mathbb{E}\left(S_{T}\left(-Z_{T}^{1}\right)^{n} \mathrm{e}^{-\rho Z_{T}^{1}}\right) \mid\right. \\
& \leq \mathbb{E}\left(\left|Z_{T}^{1}\right|^{n} \mathrm{e}^{-\rho Z_{T}^{1}}\right) \leq\left[\mathbb{E}\left(\left|Z_{T}^{1}\right|^{2 n} \mathrm{e}^{-2 \rho Z_{T}^{1}}\right)\right]^{\frac{1}{2}} .
\end{aligned}
$$

We can easily evaluate the last quantity, indeed for any Gaussian random variable $Z \sim \mathcal{N}\left(0 ; \Sigma^{2}\right)$, we have

$$
\begin{aligned}
\mathbb{E}\left(Z^{2 n} \mathrm{e}^{-2 \rho Z}\right) & =\int_{\mathbb{R}} \frac{1}{\sqrt{2 \pi \Sigma^{2}}} z^{2 n} \mathrm{e}^{-2 \rho z} \mathrm{e}^{-\frac{z^{2}}{2 \Sigma^{2}}} d z=\Sigma^{2 n} \mathrm{e}^{2 \rho^{2} \Sigma^{2}} \int_{\mathbb{R}} \frac{1}{\sqrt{2 \pi}}(v-2 \Sigma \rho)^{2 n} \mathrm{e}^{-\frac{v^{2}}{2}} d v \\
& =\Sigma^{2 n} \mathrm{e}^{2 \rho^{2} \Sigma^{2}} \sum_{i=0}^{2 n}\left(\begin{array}{c}
2 n \\
i
\end{array}\right)(-2 \Sigma \rho)^{2 n-i} \int_{\mathbb{R}} v^{i} \mathrm{e}^{-\frac{v^{2}}{2}} d v \\
& =\Sigma^{2 n} \mathrm{e}^{2 \rho^{2} \Sigma^{2}} \sum_{j=0}^{n}\left(\begin{array}{c}
2 n \\
2 j
\end{array}\right)(-2 \Sigma \rho)^{2 n-2 j} \int_{\mathbb{R}} v^{2 j} \mathrm{e}^{-\frac{v^{2}}{2}} d v \\
& =\Sigma^{2 n} \mathrm{e}^{2 \rho^{2} \Sigma^{2}} \sum_{j=0}^{n}\left(\begin{array}{c}
2 n \\
2 j
\end{array}\right)(2 \Sigma \rho)^{2 n-2 j}(2 j-1) ! ! \leq \Sigma^{2 n} \mathrm{e}^{2 \rho^{2} \Sigma^{2}}(2 n-1) ! !(1+2 \Sigma|\rho|)^{2 n}
\end{aligned}
$$

Since $|\rho|<1$, taking

$$
\Sigma^{2}:=\frac{\sigma^{2}}{k^{2}} \int_{0}^{T}\left(1-\mathrm{e}^{-k(T-s)}\right)^{2} d s
$$

we have that

$$
\left|\frac{\partial^{n}}{\partial \rho^{n}} G(\rho)\right| \leq \mathrm{e}^{\rho^{2} \Sigma^{2}} \sqrt{(2 n-1) ! !}(1+2 \Sigma)^{2 n}, \quad \forall \rho \in(-1,1),
$$

and by Lagrange Theorem

$$
\left|R_{n-1}(\rho)\right|=\left|\frac{\rho^{n}}{n !} \frac{\partial^{n}}{\partial \rho^{n}} G(\bar{\rho})\right| \leq \frac{\mathrm{e}^{\rho^{2} \Sigma^{2}}\left(|\rho \sqrt{2}|(1+2 \Sigma)^{2}\right)^{n}}{\sqrt{n !}},
$$

where $0<|\bar{\rho}|<|\rho|$. Hence the remainder $R_{n}(\rho)$ of the power series tends to zero as $n \rightarrow+\infty$ implying the real analyticity of $G(\rho)$. The product of two real-analytic functions of $\rho$ is real-analytic, and Lemma 1 implies the result.

Lastly, we recall that the Vasicek bond price is given by (see e.g. [21])

$$
P^{V}(0, r, T)=\mathrm{e}^{-\theta T-(r-\theta) \frac{1-\mathrm{e}^{-k T}}{k}+\frac{\sigma^{2}}{2 k^{2}} \int_{0}^{T}\left(1-\mathrm{e}^{-k(T-s)}\right)^{2} d s},
$$

consequently (2.11) may be rewritten as

$$
P^{d}(0, r, \lambda, T ; \rho)=P^{V}(0, r, T) \mathrm{e}^{-\frac{\rho^{2} \sigma^{2}}{2 k^{2}} \int_{0}^{T}\left(1-\mathrm{e}^{-k(T-s)}\right)^{2} d s} G(\rho) .
$$




\section{Change of numeraire}

In formula (2.17) the only non explicit factor is $G(\rho)$. For better handling of this function, we apply an appropriate change of numéraire. Let us introduce the positive $\mathcal{F}_{s}$-martingale

$$
L_{s}:=\frac{\mathbb{E}\left(\mathrm{e}^{-\int_{0}^{T} \lambda_{u} d u} \mid \mathcal{F}_{s}^{1}\right)}{\mathbb{E}\left(S_{T}\right)}=\frac{\mathrm{e}^{A(s, T)-B(s, T) \lambda_{s}}}{\mathbb{E}\left(S_{T}\right)}, \quad \Rightarrow \quad L_{0}=1
$$

where

$\delta:=\sqrt{\alpha^{2}+2 \gamma^{2}}, A(t, T):=\frac{2 \alpha \beta}{\gamma^{2}} \ln \left[\frac{2 \delta \mathrm{e}^{\alpha+\delta(T-t)}}{\delta-\alpha+(\delta+\alpha) \mathrm{e}^{\delta(T-t)}}\right], B(t, T):=\frac{2\left(\mathrm{e}^{\delta(T-t)}-1\right)}{\delta-\alpha+(\delta+\alpha) \mathrm{e}^{\delta(T-t)}}$.

The random variable $L_{T}$ defines the $T$-forward measure as $\mathbb{P}^{T}(A):=\mathbb{E}\left(L_{T} 1_{A}\right)$ (see [6] for the method, and [10] for a similar application), and we may write

$$
G(\rho)=\mathbb{E}\left(S_{T} \mathrm{e}^{-\rho Z_{T}^{1}}\right)=\mathbb{E}\left(S_{T}\right) \mathbb{E}^{\mathbb{P}^{T}}\left(\mathrm{e}^{-\rho Z_{T}^{1}}\right)=P^{C I R}(0, \lambda, T) \mathbb{E}^{\mathbb{P}^{T}}\left(\mathrm{e}^{-\rho Z_{T}^{1}}\right),
$$

where $\left.P^{C I R}(0, \lambda, T)\right):=\mathrm{e}^{A(0, T)-B(0, T) \lambda}$.

From (3.1), $d L_{s}=-\gamma L_{s} \sqrt{\lambda_{s}} B(s, T) d B_{s}^{1}$, whence by Girsanov theorem

$$
\tilde{B}_{s}^{1}:=B_{s}^{1}+\gamma \int_{0}^{s} B(u, T) \sqrt{\lambda_{u}} d u
$$

is a Brownian motion under $\mathbb{P}^{T}$ and

$$
\begin{aligned}
Z_{T}^{1} & =\frac{\sigma}{k} \int_{0}^{T}\left[1-\mathrm{e}^{-k(T-s)}\right] d B_{s}^{1}=\frac{\sigma}{k} \int_{0}^{T}\left[1-\mathrm{e}^{-k(T-s)}\right] d \tilde{B}_{s}^{1}-\gamma \frac{\sigma}{k} \int_{0}^{T}\left[1-\mathrm{e}^{-k(T-s)}\right] B(s, T) \sqrt{\lambda_{s}} d s \\
& =: \tilde{Z}_{T}^{1}-\gamma \Lambda_{T} .
\end{aligned}
$$

By setting $G^{T}(\rho):=\mathbb{E}^{\mathbb{P}^{T}}\left(\mathrm{e}^{-\rho Z_{T}^{1}}\right)=\mathbb{E}^{\mathbb{P}^{T}}\left(\mathrm{e}^{-\rho\left(\tilde{Z}_{T}^{1}-\gamma \Lambda_{T}\right)}\right)$ we may conclude

$$
P^{d}(0, r, \lambda, T ; \rho)=P^{V}(0, r, T) P^{C I R}(0, \lambda, T) \mathrm{e}^{-\frac{\rho^{2} \sigma^{2}}{2 k^{2}} \int_{0}^{T}\left[1-\mathrm{e}^{-k(T-s)}\right]^{2} d s} G^{T}(\rho),
$$

which reduces to (2.5) in the case $\rho=0$. Formula (3.3) is particularly appealing, indeed the dependence on $\rho$ in the bond price has been isolated and made more explicit. It remains only to evaluate $G^{T}(\rho)$ that we are going to approximate by a second-order Taylor polynomial, as shown in the next section.

\section{Numerical analysis: the second order approximation}

To complete the evaluation described by formula (3.3), we should compute $G^{T}(\rho)$. Here, we suggest approximating it by its second-order Taylor polynomial

$$
G_{2}^{T}(\rho):=G^{T}(0)+\frac{\partial G^{T}}{\partial \rho}(0) \rho+\frac{\partial^{2} G^{T}}{\partial \rho^{2}}(0) \frac{\rho^{2}}{2} .
$$

We opted for the second-order expansion to capture the possible nonlinearity of this function but trying to keep it simple, hoping it is enough to have good accuracy. If better accuracy were required, one could always push the expansion to some higher order.

We have $G^{T}(0)=1$, and recalling that $\tilde{Z}_{T}^{1}$ is Gaussian with zero mean and variance $\Sigma^{2}$, defined by (2.15), we obtain by differentiation

$$
\begin{aligned}
& a_{1}:=\frac{\partial G^{T}}{\partial \rho}(0)=-\mathbb{E}^{\mathbb{P}^{T}}\left(\tilde{Z}_{T}^{1}\right)+\gamma \mathbb{E}^{\mathbb{P}^{T}}\left(\Lambda_{T}\right)=\frac{\sigma \gamma}{k} \int_{0}^{T}\left(1-\mathrm{e}^{-k(T-s)}\right) \mathbb{E}^{\mathbb{P}^{T}}\left(\sqrt{\lambda_{s}}\right) B(s, T) d s \\
& a_{2}:=\frac{\partial^{2} G^{T}}{\partial \rho^{2}}(0)=\mathbb{E}^{\mathbb{P}^{T}}\left[\left(\gamma \Lambda_{T}-\tilde{Z}_{T}^{1}\right)^{2}\right]=\gamma^{2} \mathbb{E}^{\mathbb{P}^{T}}\left(\Lambda_{T}^{2}\right)+\Sigma^{2}-2 \gamma \mathbb{E}^{\mathbb{P}^{T}}\left(\tilde{Z}_{T}^{1} \Lambda_{T}\right) .
\end{aligned}
$$


The quantities $E^{\mathbb{P}^{T}}\left(\sqrt{\lambda_{s}}\right), \mathbb{E}^{\mathbb{P}^{T}}\left(\Lambda_{T}^{2}\right), \mathbb{E}^{\mathbb{P}^{T}}\left(\tilde{Z}_{T}^{1} \Lambda_{T}\right)$ cannot be computed exactly as the distributions of the random variables are unknown, hence we implement the following approximation procedures:

- In [17], the authors propose a very efficient approximation of $\mathbb{E}\left[\sqrt{\lambda_{t}}\right]$ for a CIR process, that we are going to adapt to our needs. They suggested employing a first-order Taylor polynomial approximation

$$
\mathbb{E}\left[\sqrt{\lambda_{t}}\right] \approx a+b \mathrm{e}^{-c t}:=q(t),
$$

for appropriately chosen $a, b, c$ (see Result 3.3 in [17]).

In our case, under $\mathbb{P}^{T}$, the intensity process is not a classical CIR process as it satisfies

$$
d \lambda_{s}=\alpha\left[\beta-\lambda_{s}\left(1-\gamma^{2} B(s, T)\right)\right] d s+\gamma \sqrt{\lambda_{s}} d \tilde{B}_{s}^{1} .
$$

On the other hand, $1-\gamma^{2} B(s, T)$ is a slow-varying function, so we modify the above dynamics by substituting this function with its midpoint $\bar{h}:=1-\gamma^{2} B(0, T) / 2$, to recover a standard CIR process with parameters $\bar{\alpha}=\alpha \bar{h}, \bar{\beta}=\beta / \bar{h}$. Applying the technique in [17] (that we are going to illustrate in the next subsection), we may conclude $E^{\mathbb{P}^{T}}\left(\sqrt{\lambda_{s}}\right) \approx a+b \mathrm{e}^{-c s}$, with

$$
\begin{aligned}
a & =\sqrt{\bar{\beta}-\frac{\gamma^{2}}{8 \bar{\alpha}}}, \quad b=\sqrt{\lambda}-a, \quad c=-\log \left(\frac{\Lambda-a}{b}\right), \\
\Lambda & =\sqrt{\frac{\gamma^{2}\left(1-\mathrm{e}^{-\bar{\alpha}}\right)}{4 \bar{\alpha}}\left[\frac{4 \bar{\alpha} \lambda \mathrm{e}^{-\bar{\alpha}}}{\gamma^{2}\left(1-\mathrm{e}^{-\bar{\alpha}}\right)}-1+\frac{4 \bar{\alpha} \bar{\beta}}{\gamma^{2}}+\frac{4 \bar{\alpha} \bar{\beta} / \gamma^{2}}{2\left(4 \bar{\alpha} \bar{\beta} / \gamma^{2}+\left(4 \bar{\alpha} \lambda \mathrm{e}^{-\bar{\alpha}}\right) /\left(\gamma^{2}\left(1-\mathrm{e}^{-\bar{\alpha}}\right)\right)\right.}\right]} .
\end{aligned}
$$

- To compute $\mathbb{E}^{\mathbb{P}^{T}}\left(\tilde{Z}_{T}^{1} \Lambda_{T}\right)$, we apply integration by parts $d\left(\tilde{Z}_{t}^{1} \Lambda_{t}\right)=\tilde{Z}_{t}^{1} d \Lambda_{t}+\Lambda_{t} d \tilde{Z}_{t}^{1}$. When we take the expectation, the martingale term gives no contribution, and we obtain

$$
\mathbb{E}^{\mathbb{P}^{T}}\left(\tilde{Z}_{T}^{1} \Lambda_{T}\right)=\frac{\sigma}{k} \int_{0}^{T}\left(1-\mathrm{e}^{-k(T-s)}\right) B(s, T) \mathbb{E}\left(\tilde{Z}_{s}^{1} \sqrt{\lambda_{s}}\right) d s .
$$

Approximating the process $\sqrt{\lambda_{s}}$ with its mean computed as in the previous point, we may conclude that $\mathbb{E}^{\mathbb{P}^{T}}\left(\tilde{Z}_{T}^{1} \Lambda_{T}\right) \approx 0$.

- Finally, we exploit the independence of the increments of the process $\lambda_{s}$ to compute the last expectation. Setting $f(s)=\left(1-\mathrm{e}^{-k(T-s)}\right) B(s, T)$, we have

$$
\begin{aligned}
\mathbb{E}^{\mathbb{P}^{T}}\left(\Lambda_{T}^{2}\right) & =\mathbb{E}^{\mathbb{P}^{T}}\left[\left(\frac{\sigma}{k} \int_{0}^{T} f(s) \sqrt{\lambda_{s}} d s\right)^{2}\right]=2 \frac{\sigma^{2}}{k^{2}} \int_{0}^{T} \int_{0}^{s} f(s) f(u) \mathbb{E}^{\mathbb{P}^{T}}\left(\sqrt{\lambda_{s}} \sqrt{\lambda_{u}}\right) d s d u \\
& =2 \frac{\sigma^{2}}{k^{2}} \int_{0}^{T} \int_{0}^{s} f(s) f(u)\left[\mathbb{E}^{\mathbb{P}^{T}}\left(\sqrt{\lambda_{s}}-\sqrt{\lambda_{u}}\right) \mathbb{E}^{\mathbb{P}^{T}}\left(\sqrt{\lambda_{u}}\right)-\mathbb{E}^{\mathbb{P}^{T}}\left(\lambda_{u}\right)\right] d s d u \\
& =2 \frac{\sigma^{2}}{k^{2}} \int_{0}^{T} \int_{0}^{s} f(s) f(u)\left[\mathbb{E}^{\mathbb{P}^{T}}\left(\sqrt{\lambda_{s}}\right) \mathbb{E}^{\mathbb{P}^{T}}\left(\sqrt{\lambda_{u}}\right)-\left[\mathbb{E}^{\mathbb{P}^{T}}\left(\sqrt{\lambda_{u}}\right)\right]^{2}-\mathbb{E}^{\mathbb{P}^{T}}\left(\lambda_{u}\right)\right] d s d u .
\end{aligned}
$$

The expectations of the square roots of the intensity can be approximated as before, while

$$
\mathbb{E}^{\mathbb{P}^{T}}\left(\lambda_{s}\right)=\mathrm{e}^{-\alpha \int_{0}^{s}\left(1-\gamma^{2} B(u, T)\right) d u}\left\{\lambda+\alpha \beta \int_{0}^{s} \mathrm{e}^{\alpha \int_{0}^{u}\left(1-\gamma^{2} B(v, T)\right) d v} d s\right\} .
$$




\subsection{The GO method}

Here, we briefly explain the approximation technique proposed by Grzelak and Oosterlee in [17] for a different pricing problem (hereafter GO approximation), that we adapt to our framework. Their method replaces the operator $\mathcal{A}$ given in (2.9) with

$$
\mathcal{A}^{G O}:=\rho \gamma \sigma \mathbb{E}^{Q}\left(\sqrt{\lambda_{t}}\right) \frac{\partial^{2}}{\partial r \partial \lambda} \approx \rho \gamma \sigma q(t) \frac{\partial^{2}}{\partial r \partial \lambda},
$$

obtaining the parabolic problem

$$
\left\{\begin{array}{l}
\frac{\partial u}{\partial t}(t, r, \lambda)+\left(\mathcal{L}^{0}+\mathcal{A}^{G O}\right) u(t, r, \lambda)=0 \\
u(T, r, \lambda)=1
\end{array}\right.
$$

that has a solution of the affine form $P_{G O}^{d}(t, r, \lambda, T ; \rho)=\mathrm{e}^{A(t, T)+B(t, T) r_{t}+C(t, T) \lambda_{t}}$.

As usual, plugging this expression into (4.6), one obtains the system of ODE's

$$
\begin{aligned}
& B^{\prime}(t, T)=1+\kappa B(t, T), \quad B(T, T)=0, \\
& C^{\prime}(t, T)=1+\alpha C(t, T)-\frac{\gamma^{2}}{2} C^{2}(t, T), \quad C(T, T)=0, \\
& A^{\prime}(t, T)=-\frac{\sigma^{2}}{2} B^{2}(t, T)-\kappa \theta B(t, T)-\alpha \beta C(t, T)-\rho \gamma \sigma q(t) B(t, T) C(t, T), \quad A(T, T)=0,
\end{aligned}
$$

whence

$$
\begin{aligned}
& B(t, T)=\frac{1}{\kappa}\left(\mathrm{e}^{-\kappa(T-t)}-1\right), \\
& C(t, T)=\frac{-2\left(1-\mathrm{e}^{-d(T-t)}\right)}{2 d+(\alpha+d)\left(\mathrm{e}^{-d(T-t)}-1\right)}, \quad d=\sqrt{\alpha^{2}+2 \gamma^{2}}, \\
& A(t, T)=\frac{\sigma^{2}}{2} \int_{t}^{T} B^{2}(s, T) d s+\kappa \theta \int_{t}^{T} B(s, T) d s+\alpha \beta \int_{t}^{T} C(s, T) d s+\rho \gamma \sigma \int_{t}^{T} q(s) B(s, T) C(s, T) d s .
\end{aligned}
$$

This approximation may be suggestively summarized into

$$
\begin{aligned}
P_{G O}^{d}(0, r, \lambda, T ; \rho) & =\mathrm{e}^{A(0, T)+B(0, T) r+C(0, T) \lambda} \\
& =\mathrm{e}^{B(0, T) r+\frac{\sigma^{2}}{2} \int_{0}^{T} B^{2}(s, T) d s+\kappa \theta \int_{0}^{T} B(s, T) d s \quad \text { (Vasicek Bond price) }} \\
& \times \mathrm{e}^{C(0, T) \lambda+\alpha \beta \int_{0}^{T} C(s, T) d s} \quad \text { (CIR Bond price) } \\
& \times \mathrm{e}^{\rho \gamma \sigma \int_{0}^{T} q(s) B(s, T) C(s, T) d s} \\
& =P^{V}(0, r, T) P^{C I R}(0, \lambda, T) \mathrm{e}^{\rho \gamma \sigma \int_{0}^{T} q(s) B(s, T) C(s, T) d s} .
\end{aligned}
$$

\subsection{Numerical results}

In this section, for varying $\rho$, we assess the performance of the second-order approximation

$$
\tilde{P}^{d}(0, r, \lambda, T ; \rho):=P^{V}(0, r, T) P^{C I R}(0, \lambda, T) \mathrm{e}^{-\frac{\rho^{2} \sigma^{2}}{2 k^{2}} \int_{0}^{T}\left[1-\mathrm{e}^{-k(T-s)}\right]^{2} d s} \tilde{G}_{2}^{T}(\rho),
$$

where $\tilde{G}_{2}^{T}(\rho)$ is given by

$$
\tilde{G}_{2}^{T}(\rho)=1+\tilde{a}_{1} \rho+\tilde{a}_{2} \frac{\rho^{2}}{2}
$$

To compute the $\tilde{a}_{i}, i=1,2$, we apply the procedure described at the beginning of this section. We are going to compare the results with the benchmark Monte Carlo evaluation and the GO approximation.

We chose the parameters of the intensity process as in [11] (see Table 1), which agree with those of calibrated default intensities. For the interest rate we took $r_{0}=0.01$, 
Table 1: Parameter sets for the CIR default intensity. The one-year survival probability for Set a, Set b and Set c is $96.9 \%, 90.5 \%$ and $98.7 \%$, respectively.

\begin{tabular}{|c|c|c|c|c|}
\hline & $\lambda$ & $\alpha$ & $\beta$ & $\gamma$ \\
\hline Set a & 0.03 & 0.02 & 0.161 & 0.08 \\
Set b & 0.035 & 0.35 & 0.45 & 0.15 \\
Set c & 0.01 & 0.8 & 0.02 & 0.2 \\
\hline
\end{tabular}

Table 2: Absolute error of the second order approximation with respect to the benchmark Monte Carlo prices, for maturity $T=0.5$. The length of the $95 \%$-confidence interval ranges from $7.5 \mathrm{e}-07$ to $3.9 \mathrm{e}-06$.

\begin{tabular}{r|c|c|c|c|c|c}
\hline & \multicolumn{2}{|c}{ Set a } & \multicolumn{2}{c}{ Set b } & \multicolumn{2}{c}{ Set c } \\
\hline$\rho$ & ARS & GO & ARS & GO & ARS & GO \\
\hline-0.9 & $5.7365 \mathrm{e}-07$ & $5.6891 \mathrm{e}-07$ & $3.3579 \mathrm{e}-06$ & $3.3622 \mathrm{e}-06$ & $1.1904 \mathrm{e}-06$ & $1.1665 \mathrm{e}-06$ \\
-0.6 & $9.1318 \mathrm{e}-07$ & $9.1376 \mathrm{e}-07$ & $6.0312 \mathrm{e}-06$ & $6.0336 \mathrm{e}-06$ & $1.0482 \mathrm{e}-06$ & $1.0621 \mathrm{e}-06$ \\
-0.3 & $1.0944 \mathrm{e}-07$ & $1.0941 \mathrm{e}-07$ & $2.3765 \mathrm{e}-06$ & $2.3779 \mathrm{e}-06$ & $0.3533 \mathrm{e}-06$ & $0.3465 \mathrm{e}-06$ \\
0.3 & $1.9451 \mathrm{e}-06$ & $1.9456 \mathrm{e}-06$ & $4.9327 \mathrm{e}-06$ & $4.9328 \mathrm{e}-06$ & $2.9603 \mathrm{e}-06$ & $2.9541 \mathrm{e}-06$ \\
0.6 & $7.5866 \mathrm{e}-07$ & $7.5414 \mathrm{e}-07$ & $2.3698 \mathrm{e}-06$ & $2.3635 \mathrm{e}-06$ & $0.2740 \mathrm{e}-06$ & $0.2822 \mathrm{e}-06$ \\
0.9 & $0.2135 \mathrm{e}-07$ & $0.3962 \mathrm{e}-07$ & $2.1126 \mathrm{e}-06$ & $2.0856 \mathrm{e}-06$ & $1.3778 \mathrm{e}-06$ & $1.3783 \mathrm{e}-06$ \\
\hline
\end{tabular}

$k=0.4, \theta=0.05$ and $\sigma=0.1$, and finally we considered three maturities, $T=0.5, T=1$, and $T=5$. All the algorithms were implemented in MatLab (R2019b).

We implemented the Monte Carlo method by using the Euler discretization scheme with full truncation for the intensity process $\lambda_{t}$ (see [24]) and the exact simulation of the short rate process $r_{t}$. To reduce the variance of the estimator, we used a control variates technique, taking the default-free bond price as control. In the considered cases, this shortened the length of the confidence interval by at least one order of magnitude. In our numerical experiments, we generated $M=10^{7}$ sample paths, with a time step discretization equal to $10^{-3}$ for all the maturities. The length of the $95 \%$ confidence interval ranged approximately from $1.0 \mathrm{e}-07$ to $1.0 \mathrm{e}-05 .^{1}$

In Tables $2-4$, we report the absolute errors of our implementation with respect to the Monte Carlo algorithm. The computing time to calculate one approximated price was about 0.002 seconds. Furthermore, our method achieves comparable accuracy respect to the GO approximations, which has a similar computational time of about 0.001 seconds.

The quality of our approximations appears to be satisfactory, almost uniformly in the values of the correlation parameter $\rho$, while it tends to worsen slightly for increasing maturities.

The advantage that we see in our methodology relies on the fact that a higher-order expansion might achieve better accuracy, where it is needed, while the performance of the GO approximations cannot be improved.

For the sake of completeness, we also run a basic sensitivity analysis of the approximated defaultable bond prices for the model parameters $\lambda_{0}, \theta, T, \gamma$, as the correlation varies between -0.9 and 0.9 . As expected, variations in $\rho$ do not affect the price behavior in $\lambda_{0}$ and $\theta$ much, which is roughly linear. More interesting is the effect of the maturity, $T$, and of the intensity volatility $\gamma$.

In the first case, it can be easily seen that all prices are decreasing in $T$, and below

\footnotetext{
${ }^{1}$ A Monte Carlo estimate of the defaultable bond price may be obtained directly from the representation (3.3), in which case only the intensity process (4.5) must be simulated. This reduces the computational burden of the algorithm, but results are comparable (in all the considered cases) to the MC procedure previously described.
} 
Table 3: Absolute error of the second order approximation with respect to the benchmark Monte Carlo prices, for maturity $T=1$. The lenght of the $95 \%$-confidence interval ranges from $2.0 \mathrm{e}-06$ to $1.1 \mathrm{e}-05$.

\begin{tabular}{r|c|c|c|c|c|c}
\hline & \multicolumn{2}{|c}{ Set a } & \multicolumn{2}{c}{ Set $\mathrm{b}$} & \multicolumn{2}{c}{ Set c } \\
\hline$\rho$ & ARS & GO & ARS & GO & ARS & GO \\
\hline-0.9 & $0.7728 \mathrm{e}-06$ & $0.5677 \mathrm{e}-06$ & $3.4001 \mathrm{e}-05$ & $3.4157 \mathrm{e}-05$ & $2.0549 \mathrm{e}-06$ & $1.4446 \mathrm{e}-06$ \\
-0.6 & $2.8839 \mathrm{e}-06$ & $2.9067 \mathrm{e}-06$ & $3.4866 \mathrm{e}-05$ & $3.4971 \mathrm{e}-05$ & $2.6326 \mathrm{e}-06$ & $2.9351 \mathrm{e}-06$ \\
-0.3 & $0.3118 \mathrm{e}-06$ & $0.3109 \mathrm{e}-06$ & $1.7943 \mathrm{e}-05$ & $1.8007 \mathrm{e}-05$ & $1.3155 \mathrm{e}-06$ & $1.1719 \mathrm{e}-06$ \\
0.3 & $5.4730 \mathrm{e}-06$ & $5.4977 \mathrm{e}-06$ & $1.3988 \mathrm{e}-05$ & $1.4007 \mathrm{e}-05$ & $9.5357 \mathrm{e}-06$ & $9.4207 \mathrm{e}-06$ \\
0.6 & $1.7739 \mathrm{e}-06$ & $1.5500 \mathrm{e}-06$ & $1.1658 \mathrm{e}-05$ & $1.1299 \mathrm{e}-05$ & $4.2103 \mathrm{e}-06$ & $4.1694 \mathrm{e}-06$ \\
0.9 & $0.5507 \mathrm{e}-06$ & $1.4414 \mathrm{e}-06$ & $1.6879 \mathrm{e}-05$ & $1.5483 \mathrm{e}-05$ & $1.0814 \mathrm{e}-05$ & $1.1343 \mathrm{e}-05$ \\
\hline
\end{tabular}

Table 4: Absolute error of the second order approximation with respect to the benchmark Monte Carlo prices, for maturity $T=5$. The lenght of the $95 \%$-confidence interval ranges from $1.3 \mathrm{e}-05$ to $4.3 \mathrm{e}-05$.

\begin{tabular}{r|c|c|c|c|c|c}
\hline & \multicolumn{2}{|c}{ Set a } & \multicolumn{2}{c}{ Set $\mathrm{b}$} & \multicolumn{2}{c}{ Set c } \\
\hline$\rho$ & ARS & GO & ARS & GO & ARS & GO \\
\hline-0.9 & $5.5635 \mathrm{e}-04$ & $7.2537 \mathrm{e}-04$ & $2.7751 \mathrm{e}-04$ & $1.3394 \mathrm{e}-04$ & $9.4493 \mathrm{e}-04$ & $1.4816 \mathrm{e}-03$ \\
-0.6 & $2.7842 \mathrm{e}-04$ & $2.8514 \mathrm{e}-04$ & $2.6543 \mathrm{e}-04$ & $1.2197 \mathrm{e}-04$ & $5.9211 \mathrm{e}-04$ & $7.0611 \mathrm{e}-04$ \\
-0.3 & $0.8389 \mathrm{e}-05$ & $1.4300 \mathrm{e}-05$ & $1.2988 \mathrm{e}-04$ & $0.5633 \mathrm{e}-04$ & $1.8608 \mathrm{e}-04$ & $2.1583 \mathrm{e}-04$ \\
0.3 & $1.8655 \mathrm{e}-04$ & $2.6171 \mathrm{e}-04$ & $4.0959 \mathrm{e}-04$ & $4.9280 \mathrm{e}-04$ & $0.2405 \mathrm{e}-05$ & $0.9859 \mathrm{e}-05$ \\
0.6 & $0.8296 \mathrm{e}-04$ & $6.5552 \mathrm{e}-04$ & $3.0717 \mathrm{e}-04$ & $8.7900 \mathrm{e}-04$ & $0.1987 \mathrm{e}-04$ & $3.1365 \mathrm{e}-04$ \\
0.9 & $6.8537 \mathrm{e}-04$ & $1.3782 \mathrm{e}-03$ & $3.5605 \mathrm{e}-04$ & $1.4314 \mathrm{e}-03$ & $6.3288 \mathrm{e}-04$ & $8.5577 \mathrm{e}-04$ \\
\hline
\end{tabular}

the default-free price, and the more negative the correlation is, the lower the price. The latter is a very reasonable effect: higher intensity should imply lower prices.

The behavior of the price in $\gamma$ is more articulate. As a matter of fact, a visual analysis of the price curves shows that positive correlation amplifies the usual increasing effect of volatility upon bond prices, while the interplay between $r$ and $\lambda$ may revert this behavior for large negative values of $\rho$, lowering the bond price.

\section{References}

[1] Aguilar J. P., On expansions for the Black-Scholes prices and hedge parameters, J. Math. Anal. Appl., 458, 973-989 (2019). MR3979141

[2] Antonelli F., Scarlatti S., Pricing Options under stochastic volatility: a power series approach, Finance and Stochastics, 13, 269-303 (2009). MR2482054

[3] Antonelli F., Ramponi A., Scarlatti S., Exchange option pricing under stochastic volatility: a correlation expansion, Review of Deriv. Research, 13, 45-73 (2010). MR2482054

[4] Antonelli F., Ramponi A., Scarlatti S., CVA and vulnerable options by correlation expansion, Annals of Operations Research, https://doi.org/10.1007/s10479-019-03367-z, 1-27 (2019). MR4239756

[5] Ballestra T., Pacelli G., Radi D.,Modeling CDS spreads: A comparison of some hybrid approaches, Journal of Empirical Finance, 57,107-124 (2020)

[6] Bjork T., Arbitrage Theory in Continuous Time, Oxford University Press (2009).

[7] Bielecki T. R., Rutkowski M., Credit Risk: Modeling, Valuation and Hedging, Springer Finance Series (2004). MR1869476

[8] Bielecki T. R., Jeanblanc M., Rutkowski M., Valuation and Hedging of Credit Derivatives, Lecture notes CIMPA- UNESCO Morocco School (2009). MR1869476 
[9] Brigo D., Francischello M., Pallavicini A., Analysis of Nonlinear Valuation Equations under Credit and Funding Effects, In Innovations in Derivatives Markets, Springer Proceedings In Mathematics \& Statistics, 165, 37-52 (2016).

[10] Brigo D., Vrins F., Disentangling wrong-way risk: pricing credit valuation adjustment via change of measures, European Journal of Operational Research, 269, 1154-1164 (2018). MR3797968

[11] Brigo D., Hvolby T., Vrins F., Wrong-Way Risk adjusted exposure: Analytical Approximations for Options in Default Intensity Models, In Innovations in Insurance, Risk and Asset Management, WSPC Proceedings (2018). MR3797968

[12] Cathcart L., El-Jahel L.,Pricing defaultable bonds: a middle-way approach between structural and reduced-form models, Quantitative Finance, 6, 243-253 (2006) MR2241107

[13] Cetin U., Jarrow R., Protter P.,Yildirim Y., Modeling Credit Risk with Partial Information, Annals of Appl.Prob.,14,1167-1178 (2004). MR2071419

[14] Christoffersen, P., Jacobs, K., and Mimouni, K. Volatility dynamics for the S\&P 500: evidence from realized volatility, daily returns, and option prices, The Review of Financial Studies, 23(8):3141-3189 (2010). MR3948449

[15] Duffie D.,Lando D.,Term structures of credit spreads with incomplete accounting information. Econometrica, 69, 633-664 (2001). MR1828538

[16] Duffie D., Singleton K. J., Modeling term structures of defaultable bonds, Review Financial Studies, 12, 687-720 (1999).

[17] Grzelak L. A., Oosterlee, C. W., On the Heston model with stochastic interest rates, SIAM Journal on Financial Mathematics, 2, 1, 255-286 (2011). MR2781176

[18] Kaeck, A. and Alexander, C, Volatility dynamics for the S\&P 500: further evidence from non-affine, multi-factor jump diffusions, Journal of Banking \& Finance, 36, 3110-3121 (2012).

[19] Jarrow R., Turnbull S., Pricing derivatives on financial securities subject to credit risk, Journal of Finance 50, 53-85 (1995).

[20] Jiang T., Qian X., Yuan G. X., Partial differential equation pricing method for double-name credit-linked notes with counterparty risk in a reduced-form model with common shocks, J. Math. Anal. Appl., 451, 1, 209-228 (2017). MR3619235

[21] Lamberton D., Lapeyre B. Introduction to Stochastic Calculus Applied to Finance,Chapman \& Hall (1998). MR1422250

[22] Lando D., On Cox Processes and Credit Risky Securities, Review of Derivatives Research, 2, 99-120 (1998).

[23] Lando D., Credit Risk Modeling, Princeton University Press (2004).

[24] Lord R., Koekkoek R., Van Dijk D., A comparison of biased simulation schemes for the stochastic volatility models, Quantitative Finance, 10 (2), 177-194 (2010). MR2642962

[25] Merton, R.C., On the pricing of corporate debt: the risk structure of interest rates, Journal of Finance, 2, 449-470 (1974).

[26] Madan D.,Unal H.,Pricing the risks of default, Review of Derivatives Research, 2, 121-160, (1998).

[27] Russo, V., Giacometti, R., Fabozzi, F. J., Market implied volatilities for defaultable bonds, Annals of Operations Research, 275 (2), 669-683 (2019). MR3923562

Acknowledgments. We thank the anonymous Referee for the careful reading of the paper. 